\title{
Dielectric investigations of polycrystalline samarium bismuth ferrite ceramic
}

\author{
E. Palaimiene, J. Macutkevic, D. V. Karpinsky, A. L. Kholkin, and J. Banys
}

Citation: Appl. Phys. Lett. 106, 012906 (2015); doi: 10.1063/1.4905344

View online: http://dx.doi.org/10.1063/1.4905344

View Table of Contents: http://aip.scitation.org/toc/apl/106/1

Published by the American Institute of Physics

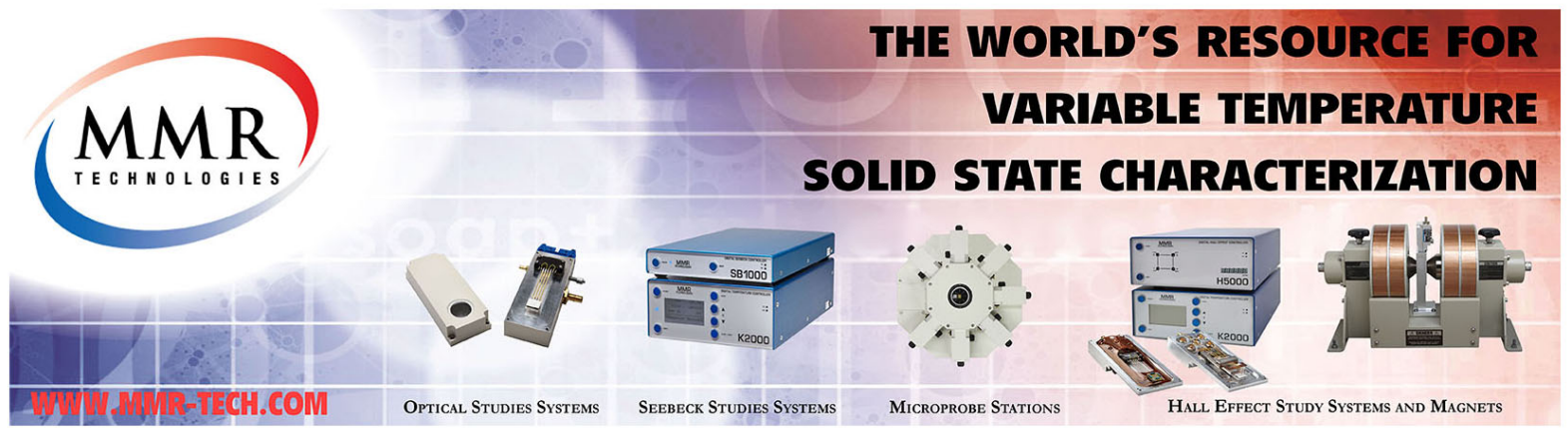




\title{
Dielectric investigations of polycrystalline samarium bismuth ferrite ceramic
}

\author{
E. Palaimiene, ${ }^{1}$ J. Macutkevic, ${ }^{1}$ D. V. Karpinsky, ${ }^{2}$ A. L. Kholkin, ${ }^{2}$ and J. Banys ${ }^{1}$ \\ ${ }^{1}$ Faculty of Physics, Vilnius University, Sauletekio Ave. 9, III b., LT 10222 Vilnius, Lithuania \\ ${ }^{2}$ Department of Ceramics and Glass Engineering and CICECO, University of Aveiro, P 3810193 Aveiro, \\ Portugal
}

(Received 18 November 2014; accepted 19 December 2014; published online 6 January 2015)

\begin{abstract}
Results of broadband dielectric investigations of samarium doped bismuth ferrite ceramics are presented in wide temperature range $(20800 \mathrm{~K})$. At temperatures higher than $400 \mathrm{~K}$, the dielectric properties of samarium bismuth ferrite ceramics are governed by Maxwell-Wagner relaxation and electrical conductivity. The DC conductivity increases and activation energy decreases with samarium concentration. In samarium doped bismuth ferrite, the ferroelectric phase transition temperature decreases with samarium concentration and finally no ferroelectric order is observed at $\mathrm{x}=0.2$. At lower temperatures, the dielectric properties of ferroelectric samarium doped bismuth ferrite are governed by ferroelectric domains dynamics. Ceramics with $\mathrm{x}=0.2$ exhibit the relaxorlike behaviour. (C) 2015 AIP Publishing LLC. [http://dx.doi.org/10.1063/1.4905344]
\end{abstract}

Multiferroic materials are currently of great interest because of its potential applications in the field of spintronics and novel multi-state memories. ${ }^{1}$ Bismuth ferrite, $\mathrm{BiFeO}_{3}$ (BFO), is one of few materials exhibiting ferroelectric (FE) and antiferromagnetic (AFM) ordering above room temperature. ${ }^{2}$ Moreover, BFO perhaps is the unique material with very high temperatures of ferroelectric-paraelectric $\left(\mathrm{T}_{\mathrm{C}} \approx 1100 \mathrm{~K}\right)$ and antiferromagnetic-paramagnetic $\left(\mathrm{T}_{\mathrm{N}} \approx 643 \mathrm{~K}\right)$ transitions. However, the BFO drawbacks are high leakage current and dielectric losses. ${ }^{3}$ Moreover, BFO could not be used for device applications due to its weak ferromagnetism. ${ }^{4}$ In order to improve $\mathrm{BFO}$ multiferroic performance, the part of $\mathrm{Bi}$ ions was successfully substituted by rare earth element ( $\mathrm{Sm}, \mathrm{Dy}, \mathrm{La}$, and $\mathrm{Gd}){ }^{5}$ For example, it was observed an increase of spontaneous magnetization and magnetoelectric coupling in samarium modified bismuth ferrite (i.e., $\mathrm{Bi}_{1}{ }_{x} \mathrm{Sm}_{\mathrm{x}} \mathrm{FeO}_{3}$ ). ${ }^{6,7}$

Single-phase bulk $\mathrm{BiFeO}_{3}$ exhibits a rhombohedral structure with space group $R 3 c$, where all ions are displaced along the $(111)_{c}$ direction of the ideal perovskite lattice and the oxygen octahedra surrounding the transition-metal cations are rotated alternately clockwise and counterclockwise about this $(111)_{c}$ direction. ${ }^{8}$ The dielectric properties of pure BFO are caused by phonon mode softening and at higher temperatures by Maxwell-Wagner relaxation. ${ }^{9}$ In contrast, the XRD pattern obtained for the $\mathrm{x}=0.1$ of in $\mathrm{Bi}_{1}{ }_{\mathrm{x}} \mathrm{Sm}_{\mathrm{x}} \mathrm{FeO}_{3}$ system was successfully indexed in rhombohedral system, for $\mathrm{x}=0.2 \mathrm{com}$ pounds an orthorhombic symmetry was detected, while compound $\mathrm{x}=0.15$ demonstrates a more complex behaviour. ${ }^{7}$ For BFO doped with various elements (La, Nd, Sm, and Gd), generally, the ferroelectric phase transition temperature decreases with addition concentration ${ }^{10}$ and at higher substitutions concentration ferroelectric order vanishes, for example, in samarium substituted BFO polar to non-polar transition is observed at $\mathrm{x}=0.2$. $^{7}$ Moreover, the antiferroelectric phase transition is usually observed at intermediate substitution concentrations in doped BFO. ${ }^{10,11}$ However, in doped materials, the ferroelectric phase transition smearing scenario can be various and relaxor or dipolar glass phase can be appear, for such investigations broadband dielectric spectroscopy is very useful. $^{12}$
The aim of the performed study is to investigate broadband dielectric properties of samarium bismuth ferrite, $\mathrm{Bi}_{1}{ }_{x} \mathrm{Sm}_{x} \mathrm{FeO}_{3}(x=0.1,0.15$, and 0.2$)$, ceramics in wide temperature range $(20800 \mathrm{~K})$.

The $\mathrm{Bi}_{1}{ }_{x} \mathrm{Sm}_{\mathrm{x}} \mathrm{FeO}_{3}(\mathrm{x}=0.1,0.15$, and 0.2$)$ ceramics were grown by a two-stage solid-state reaction method using the oxides $\mathrm{Bi}_{2} \mathrm{O}_{3}, \mathrm{Sm}_{2} \mathrm{O}_{3}$, and $\mathrm{Fe}_{2} \mathrm{O}_{3}$ and synthesis at high temperature. $^{7}$ Measurements of the complex permittivity $\varepsilon^{*}=\varepsilon^{\prime}-\mathrm{i} \varepsilon^{\prime \prime}$ were performed in wide $20 \mathrm{~K} 800 \mathrm{~K}$ temperature range at $20 \mathrm{~Hz} 42 \mathrm{GHz}$ frequencies. A LCR-meter HP4284 was used to measure capacitance and loss tangent of samples in $20 \mathrm{~Hz} 1 \mathrm{MHz}$ frequency range and model of flat dielectric capacitor was used to obtain a complex dielectric permittivity. Measurements of complex transmission and reflection coefficients were performed using Agilent 8714ET network analyzer in $1 \mathrm{MHz} 3 \mathrm{GHz}$ frequency range. In this case, a multimode capacitor model was used to obtain complex dielectric permittivity. Measurements in $1046 \mathrm{GHz}$ frequency range were performed using scalar network analyser R2400 produced by "Elmika" company by placing the thin dielectric rod in the centre of a waveguide and monitoring the reflectivity and transmission coefficients. All measurements were performed in cooling cycle with temperature change rate of about $1 \mathrm{~K} / \mathrm{min}$. Cylindrically shaped samples where used for measurements. Dimensions of the samples were with different radius: in $20 \mathrm{~Hz} 1 \mathrm{MHz}$ frequency range, the radius of samples was about $3 \mathrm{~mm}$, in $1 \mathrm{MHz} 3 \mathrm{GHz}$ frequency range it was about $0.5 \mathrm{~mm}$, while in $1046 \mathrm{GHz}$ frequency range it was only about $0.1 \mathrm{~mm}$. Silver paste was used for contacts.

Results of the dielectric measurements are presented in Fig. 1. At lower frequencies and higher temperatures (above $400 \mathrm{~K}$ ), the increase in both real and imaginary parts of complex dielectric permittivity with temperature is caused by electrical conductivity effects. At temperatures higher than $400 \mathrm{~K}$, dielectric anomalies are caused by Maxwell-Wagner polarization. ${ }^{9}$ For ceramics with $\mathrm{x}=0.15$, a frequency independent dielectric anomaly is observed close to $\mathrm{T}=356 \mathrm{~K}$. The anomaly is related with the antiferroelectric phase transition, similarly as in other BFO related materials. ${ }^{10,11}$ At 

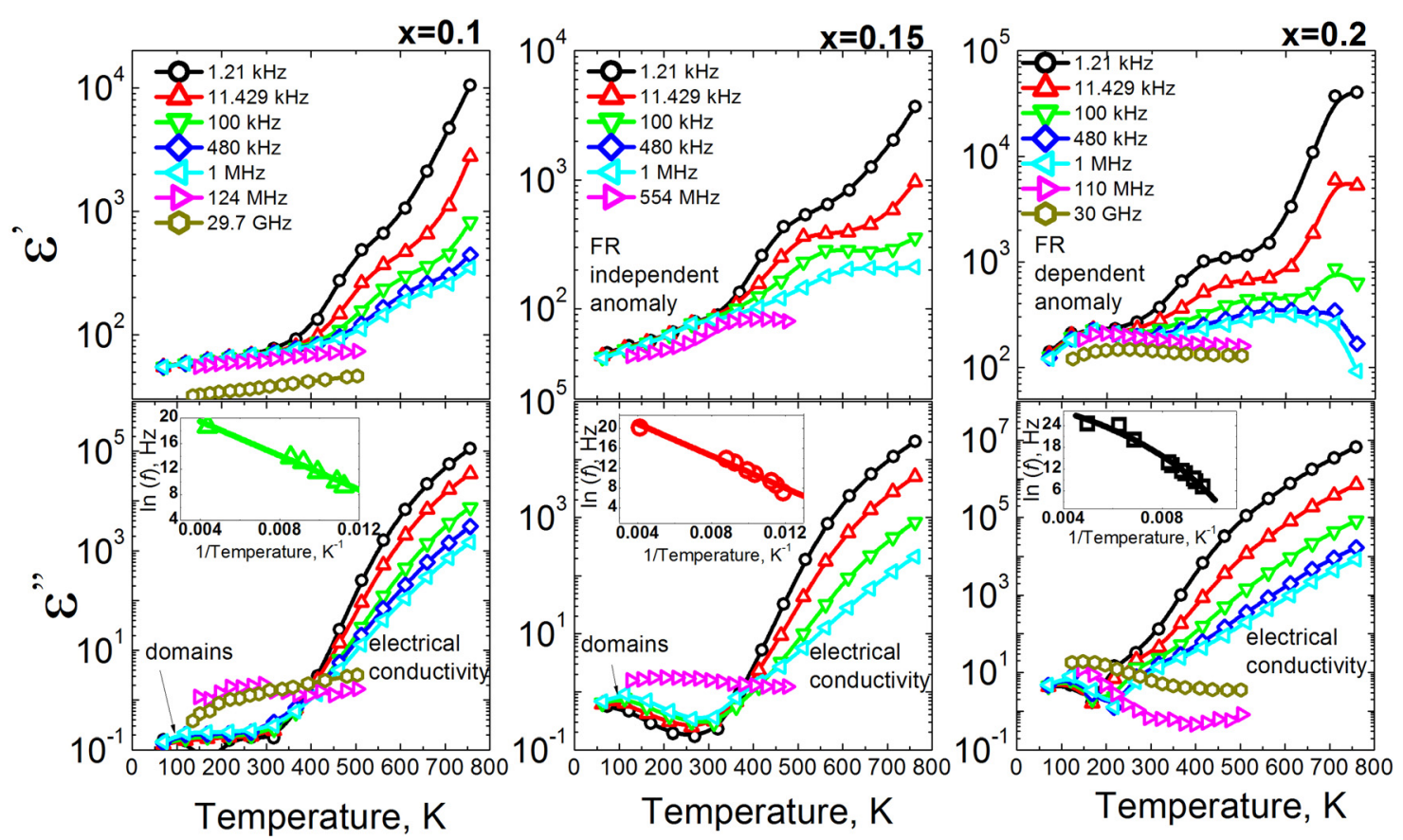

FIG. 1. Temperature dependence of the real real $\left(\varepsilon^{\prime}\right)$ and imaginary $\left(\varepsilon^{\prime \prime}\right)$ parts of complex dielectric permittivity of $\mathrm{Bi}_{1}{ }_{\mathrm{x}} \mathrm{Sm}_{\mathrm{x}} \mathrm{FeO}_{3} \mathrm{ceramics}$ at different fre quencies. The inset shows temperature dependence the maximum of imaginary part versus frequency.

low temperatures (below $200 \mathrm{~K}$ ), the maximum position of imaginary part of complex dielectric permittivity is frequency dependent (inset in Fig. 1). For ceramics with $\mathrm{x}=0.1$ and 0.15 , the dependence fits the Arrhenius law $\nu=\nu_{\infty}$ $\exp \left(-U_{V F} / T\right)$. The parameters of Arrhenius law fit are $\nu_{0}=5.8 \times 10^{10} \mathrm{~Hz}, U_{V F}=1335 \mathrm{~K}$ for $\mathrm{x}=0.1$ and $\nu_{0}=8.0$ $\times 10^{11} \mathrm{~Hz}, U_{V F}=1618 \mathrm{~K}$ for $\mathrm{x}=0.15$. The dependence fits the Vogel-Fulcher law $\nu=\nu_{\infty} \exp \left(-U_{V F} /\left[T-T_{\mathrm{f}}\right]\right)$. Obtained parameters are $T_{\mathrm{f}}=57.8 \mathrm{~K}, \nu_{0}=9.9 \times 10^{14} \mathrm{~Hz}$, and $U_{V F}$ $=1271 \mathrm{~K}$ for $\mathrm{x}=0.2$. For compounds $\mathrm{x}=0.1$ and $\mathrm{x}=0.15$ where ferroelectric order is not vanish, ${ }^{7}$ the dielectric dispersion at very low temperatures should be caused by ferroelectric domains dynamics. However, at $\mathrm{x}=0.2$, no ferroelectric order was detected by piezoelectric force microscopy ${ }^{7}$ and ferroelectric domains dynamics cannot be the origin of dielectric dispersion. Thus, the dielectric dispersion in this material should be investigated more in detail.

The frequency dependencies of the real and imaginary parts of complex dielectric permittivity of $\mathrm{Bi}_{1}{ }_{x} \mathrm{Sm}_{x} \mathrm{FeO}_{3}$ ceramics ( $\mathrm{x}=0.1$ and $\mathrm{x}=0.2)$ at lower temperatures (below $260 \mathrm{~K})$ are presented in Fig. 2. For all ceramics under study, the main dielectric dispersion occurs between several hundred megahertz and several hundred gigahertz. The dielectric dispersion for $\mathrm{x}=0.1$ and $\mathrm{x}=0.15$ was fitted with the modified Debye formula

$$
\varepsilon^{*}=\varepsilon^{\prime}-i \varepsilon^{\prime \prime}=\varepsilon_{\infty}+\frac{\Delta \varepsilon}{1+i \omega \tau}-\frac{i \sigma}{\varepsilon_{0} \omega}
$$

where $\varepsilon_{\infty}$ the dielectric permittivity when $\omega \rightarrow \infty, \Delta \varepsilon$ the dielectric strength, $\tau$ the mean relaxation time, $\sigma$ the frequency dependent conductivity, and $\varepsilon_{0}$ the vacuum dielectric permittivity. Solid lines represent a best fit (Fig. 2). The obtained temperature dependence of the mean relaxation times $\mathrm{Bi}_{0.9} \mathrm{Sm}_{0.1} \mathrm{FeO}_{3}$ ceramics is plotted in the inset of Fig. 3. Solid lines represent a fit of Arrhenius law
$\tau=\tau_{\mathrm{D}} \exp \left(-E_{\mathrm{A}} / k T\right)$. The values of Arrhenius fit law parameters are $E_{\mathrm{A}}=0.013 \mathrm{eV}, \tau_{\mathrm{D}}=4.73 \times 10^{11} \mathrm{~s}$ for $\mathrm{x}=0.1$ and $E_{\mathrm{A}}=0.0014 \mathrm{eV}, \tau_{\mathrm{D}}=2.45 \times 10^{11} \mathrm{~s}$ for $\mathrm{x}=0.15$. Arrhenius behaviour of relaxation time is typical for ferroelectric domains dynamic. ${ }^{13}$ Therefore, the dielectric dispersion in $\mathrm{Bi}_{1}{ }_{\mathrm{x}} \mathrm{Sm}_{\mathrm{x}} \mathrm{FeO}_{3}$ ceramics with $\mathrm{x}=0.1$ and $\mathrm{x}=0.15$ at microwaves is caused by ferroelectric domains dynamics. At higher samarium concentrations, dielectric dispersion demonstrates more complex behaviour $(x=0.2)$; there the dielectric dispersion cannot be described by single Debye formula (Eq. (1)) (Fig. 2). Therefore, general approach has to be used for determination of the distribution function of relaxation times $f(\tau)$ by solving Fredholm integral equation

$$
\varepsilon^{*}(v)=\varepsilon_{\infty}+\Delta \varepsilon \int_{\infty}^{\infty} \frac{f(\tau) d \ln \tau}{1+i \omega \tau},
$$

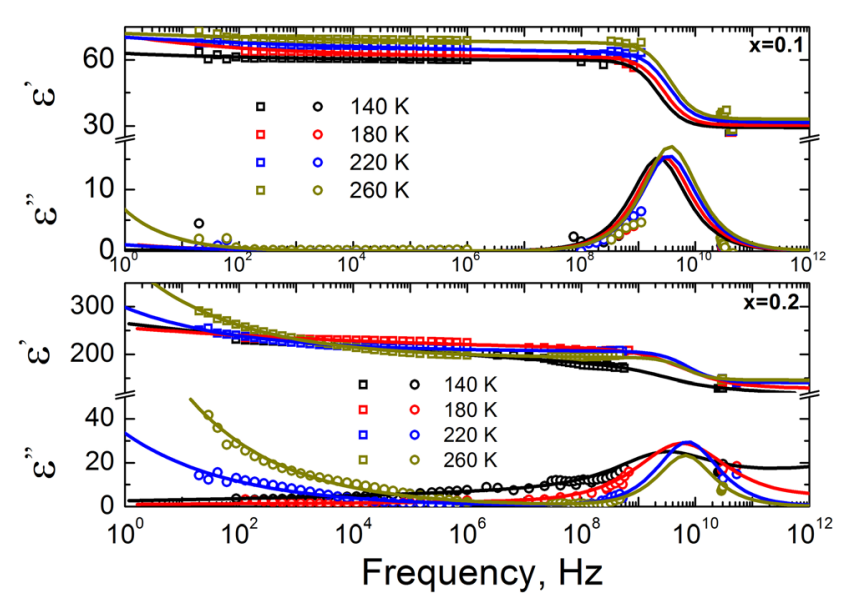

FIG. 2. Frequency dependence of the real $\varepsilon^{\prime}$ and imaginary $\varepsilon^{\prime \prime}$ parts of complex dielectric permittivity of $\mathrm{Bi}_{1}{ }_{x} \mathrm{Sm}_{\mathrm{x}} \mathrm{FeO}_{3}$ ceramics at different temperatures. 


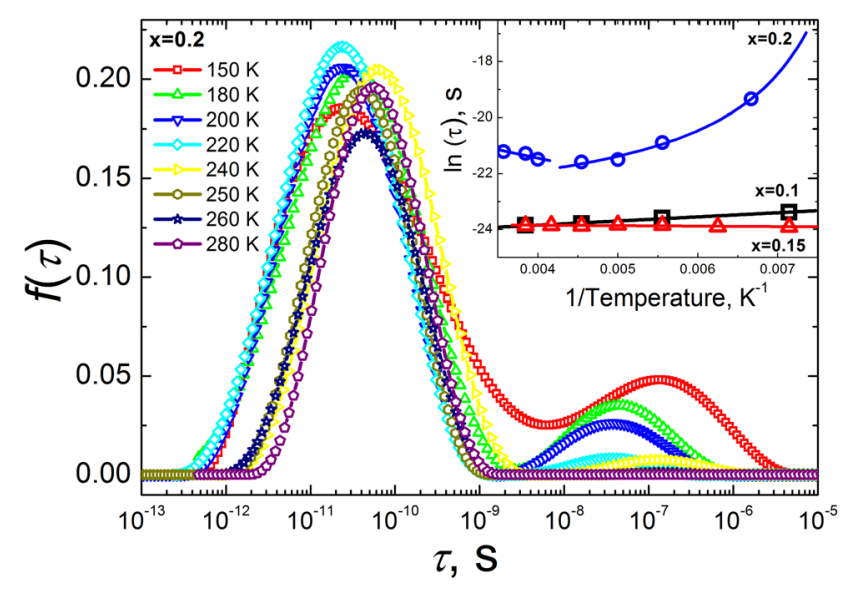

FIG. 3. Distribution of relaxation times $\mathrm{f}(\tau)$ of $\mathrm{Bi}_{0.8} \mathrm{Sm}_{0.2} \mathrm{FeO}_{3}$ ceramics cal culated at different temperatures. The inset shows temperature dependence of maximum relaxation time for $\mathrm{Bi}_{1}{ }_{x} \mathrm{Sm}_{\mathrm{x}} \mathrm{FeO}_{3}$ ceramics.

with the normalization condition

$$
\int_{\infty}^{\infty} f(\tau) d(\ln \tau)=1
$$

The Tikhonov regularization method has been used. This method and calculation technique are described in detail elsewhere. ${ }^{14}$ The distributions of relaxation times are presented in Fig. 3. At low temperatures (below $230 \mathrm{~K}$ ), the longest relaxation times $(0.1$ of maximum $f(\tau)$ was chosen for definition) were obtained from the distribution function and fitted with the Vogel-Fulcher law (see inset Fig. 3) $\tau(T)=\tau_{\mathrm{D}}$ $\exp \left(E_{B} / \mathrm{k}\left(T-T_{\mathrm{f}}\right)\right)$. Obtained values are $E_{\mathrm{B}}=0.01 \mathrm{eV}$, $\tau_{\mathrm{D}}=1.29 \times 10^{10} \mathrm{~s}$, and $T_{\mathrm{f}}=115 \mathrm{~K}$. At higher temperatures (above $230 \mathrm{~K}$ ), the dielectric dispersion is symmetric and the longest relaxation time follows the Arrhenius law. In this case, the activation energy is equal to $E_{\mathrm{B}}=0.052 \mathrm{eV}$, $\tau_{\mathrm{D}}=5.43 \times 10^{9} \mathrm{~s}$. The frequency dependent maximum of complex dielectric permittivity in all frequency range, broad dielectric dispersion, and two maxima in distribution of relaxation times is typical for ferroelectric relaxors. ${ }^{15}$

At higher temperatures (above $400 \mathrm{~K}$ ), the electrical conductivity dominates in the properties of ceramics. The electric conductivity $\sigma^{*}$ has been calculated according to the equation: $\sigma^{*}=\mathrm{i} \varepsilon^{*} \varepsilon_{0} \omega$. In Fig. 4(a) is presented the frequency dependency of conductivity at different temperatures for $\mathrm{Bi}_{1}{ }_{\mathrm{x}} \mathrm{Sm}_{\mathrm{x}} \mathrm{FeO}_{3}$ ceramics (with $\mathrm{x}=0.2$ ). The conductivity follows the Almond-West power law ${ }^{16} \sigma(\omega)=\sigma_{\mathrm{DC}}+\mathrm{A} \omega \omega^{\mathrm{s}}$, where $\sigma_{\mathrm{DC}}$ is the $\mathrm{DC}$ conductivity and $\mathrm{A} \omega{ }^{\mathrm{s}}$ is the $\mathrm{AC}$ conductivity. From these dependencies, it is possible to determine DC conductivity. With increasing Sm concentration, the DC conductivity can be determined at lower temperatures (in our measurements frequency range), for example, when $\mathrm{x}=0.2$ the DC conductivity can be determined even at $\mathrm{T}=350 \mathrm{~K}$. The values of DC conductivity for ceramics with $\mathrm{x}=0.1$ and $\mathrm{x}=0.15$ are similar, while for ceramics $\mathrm{x}=0.2$ it is substantially higher. From obtained values of $\sigma_{\mathrm{DC}}$ at different temperatures, one can calculate activation energy $\left(E_{\mathrm{A}}\right)$ and pre-exponential factor $\sigma_{0}$ of the conductivity according to the Arrhenius law $\sigma_{\mathrm{DC}}=\sigma_{0} \exp \left(-E_{\mathrm{A}} / k T\right)$ (Fig. 4(b)). The value of activation energy for $\mathrm{Bi}_{1}{ }_{\mathrm{x}} \mathrm{Sm}_{\mathrm{x}} \mathrm{FeO}_{3}$ ceramics is $E_{\mathrm{A}}=0.95 \mathrm{~K}$ and $\sigma_{0}=278662 \mathrm{Sm}^{1}$ (for $\mathrm{x}=0.1$ ); $E_{\mathrm{A}}=0.70 \mathrm{eV}$ and

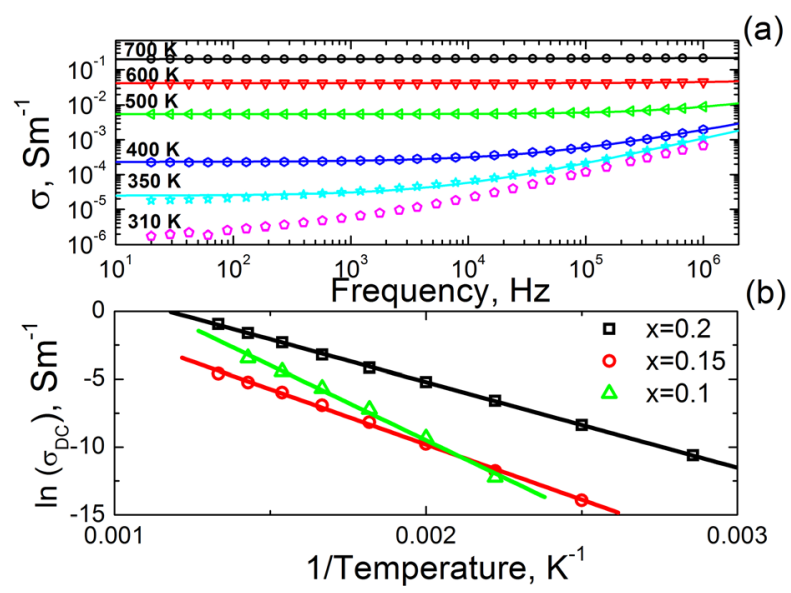

FIG. 4. (a) Frequency dependencies of conductivity at different temperatures for $\mathrm{Bi}_{0.8} \mathrm{Sm}_{0.2} \mathrm{FeO}_{3}$ ceramics. (b) The $1 / \mathrm{T}$ dependence of $\sigma_{\mathrm{DC}}$ for $\mathrm{Bi}_{1} \quad \mathrm{Sm}_{\mathrm{x}} \mathrm{FeO}_{3}$ ceramics.

$\sigma_{0}=636 \mathrm{Sm}^{1}$ (for $\mathrm{x}=0.15$ ); and $E_{\mathrm{A}}=0.54 \mathrm{eV}$ and $\sigma_{0}$ $=1605 \mathrm{~S} \mathrm{~m}^{1}$ (for $\mathrm{x}=0.2$ ). In $\mathrm{Bi}_{1}{ }_{\mathrm{x}} \mathrm{Sm}_{\mathrm{x}} \mathrm{FeO}_{3}$ ceramics, the activation energy decreases when $\mathrm{Sm}$ concentration increases. In order to get more inside conductivity phenomena, a specific resistance formalism was employed. ${ }^{17}$ The complex specific resistance has been calculated according to the formula

$$
\rho^{*}=\frac{1}{\sigma^{*}}=\frac{\varepsilon^{\prime 2}-i \varepsilon^{\prime 2}}{\varepsilon_{0} \omega\left(\varepsilon^{\prime 2}+\varepsilon^{\prime \prime 2}\right)} .
$$

The complex specific resistance was modelled as infinite chain of $R C$ circuits connected in series

$$
\rho^{*}(\nu)=\rho_{\infty}+\Delta \rho \int_{\infty}^{\infty} \frac{f(\tau) d \ln \tau}{1+i \omega \tau}
$$

where $\tau=\mathrm{RC}$. Distributions of relaxation times $f(\tau)$ were calculated at different temperatures (Fig. 5(a)).

On cooling, the distributions of relaxations times become broader and more asymmetric. Finally, at low temperatures, a second contribution to electrical conductivity appears. From distributions of relaxations time, the most probable relaxation time and the longest relaxation time

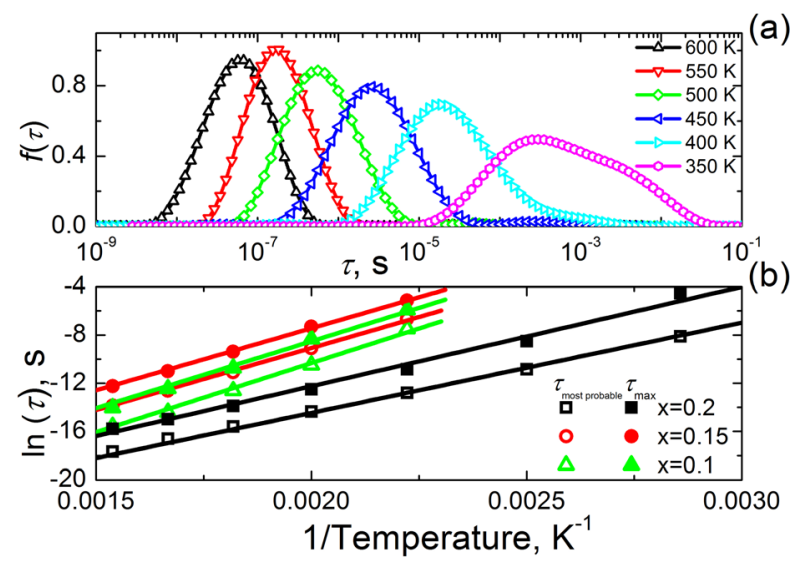

FIG. 5. (a) Distribution of relaxation times $\mathrm{f}(\tau)$ of $\mathrm{Bi}_{0.8} \mathrm{Sm}_{0.2} \mathrm{FeO}_{3}$ ceramics calculated at different temperatures. (b) Temperature dependence of relaxa tion time for $\mathrm{Bi}_{1}{ }_{x} \mathrm{Sm}_{\mathrm{x}} \mathrm{FeO}_{3}$ ceramics. Solid lines are the best fit according to the Arrhenius law. 
TABLE I. The best fitted parameters to the Arrhenius law $\tau \quad \tau_{0} \exp \left(U_{\mathrm{A}} / T\right)$.

\begin{tabular}{lccccccc}
\hline \hline & \multicolumn{2}{c}{$\mathrm{Bi}_{0.9} \mathrm{Sm}_{0.1} \mathrm{FeO}_{3}$} & & \multicolumn{2}{c}{$\mathrm{Bi}_{0.85} \mathrm{Sm}_{0.15} \mathrm{FeO}_{3}$} & & \multicolumn{2}{c}{$\mathrm{Bi}_{0.8} \mathrm{Sm}_{0.2} \mathrm{FeO}_{3}$} \\
\cline { 2 - 3 } & $f(\tau)_{\max }$ & $f(\tau)_{\text {most probable }}$ & & $f(\tau)_{\max }$ & $f(\tau)_{\text {most probable }}$ & & $f(\tau)_{\text {max }}$ \\
\hline$U_{\mathrm{A}}(\mathrm{K})$ & 11094 & 11466 & 10292 & 10292 & 8252 & 7492 \\
$\tau_{0}(\mathrm{ps})$ & 0.046 & 0.0037 & 0.68 & 0.13 & 0.32 & 0.16 \\
\hline \hline
\end{tabular}

(0.1 of maximum $f(\tau)$ was chosen for definition) were calculated (Fig. 5(b)). Both relaxation times are temperature dependent and fit the Arrhenius law. Obtained parameters are presented in Table I. The activation energy decreases with Sm concentration (Fig. 5(b)) in good agreement with DC conductivity data (Fig. 4(b)). Moreover, for ceramics with $\mathrm{x}=0.1$ and $\mathrm{x}=0.2$, the values of activation energy for most probable and longest relaxation time are different, indicating two different mechanisms in electrical transport. These contributions to electrical conductivity are related with electrical transport in grains and between grains (grain boundary effects). In BFO, the main contribution to electric conductivity appears due to oxygen vacancies transport. ${ }^{18}$ The values of activation energy presented in Table I are typical for double ionized oxygen vacancies relates to deep energy level. ${ }^{19}$

In samarium doped bismuth ferrite, the ferroelectric phase transition temperature decreases with samarium concentration and finally no ferroelectric order is observed at $\mathrm{x}=0.2$, where the relaxor-like behaviour was observed. For middle concentration $(\mathrm{x}=0.15)$, the antiferroelectric phase transition was observed. At higher temperatures (above $400 \mathrm{~K}$ ), the dielectric properties of samarium bismuth ferrite ceramics are governed by Maxwell-Wagner relaxation and electrical conductivity. The DC conductivity increases and activation energy decreases with samarium concentration, specially at higher samarium concentrations.

The authors gratefully acknowledge the financial support for this work by the European Social Fund under the Global Grant measure (Project VP1-3.1-SMM-07-K-03-011).
${ }^{1}$ W. Eerenstein, N. D. Mathur, and J. F. Scott, Nature 442, 759 (2006).

${ }^{2}$ G. Catalan and J. F. Scott, Adv. Mater. 21, 2463 (2009).

${ }^{3} \mathrm{Y} . \mathrm{Li}, \mathrm{Z} . \mathrm{Hu}$, and J. Chu, Ferroelectrics Characterization and Modeling (InTech, 2011).

${ }^{4}$ F. Kubel and H. Schmidt, Acta Crystallogr., Sect. B: Struct. Sci. 46, 698 (1990).

${ }^{5}$ V. A. Khomcenko, V. V. Shvartsman, P. Borisov, W. Kleeman, D. A. Kiselev, I. K. Bdikin, J. M. Viera, and A. L. Kholkin, Acta Mater. 57, 5137 (2009).

${ }^{6}$ S. Pattanayak, R. N. P. Chandhary, and P. R. Das, Electron. Mater. Lett. 10, 165 (2014).

${ }^{7}$ V. A. Khomchenko, J. A. Paixao, V. V. Shvartsman, P. Borisov, W. Kleemann, D. V. Karpinsky, and A. L. Kholkin, Scr. Mater. 62, 238241 (2010).

${ }^{8}$ B. Ruette, S. Zvyagin, A. P. Pyatakov, A. Bush, J. F. Li, V. I. Belotelov, A. K. Zvezdin, and D. Viehland, Phys. Rev. B 69, 064114 (2004).

${ }^{9}$ S. Kamba, D. Nuzhnyy, M. Savinov, J. Sebek, J. Petzelt, J. Prokleska, R. Haumont, and J. Kreisel, Phys. Rev. B 75, 024403 (2007).

${ }^{10}$ S. Karimi, I. M. Reaney, Y. Han, J. Pokorny, and I. Sterianou, J. Mater. Sci. 44, 5102 (2009).

${ }^{11}$ V. Goian, S. Kamba, S. Greicius, D. Nuzhnyy, S. Karimi, and I. M. Reaney, J. Appl. Phys. 110, 074112 (2011).

${ }^{12}$ J. Macutkevic, J. Banys, R. Grigalaitis, and Yu. Vysochanskii, Phys. Rev. B 78, 064101 (2008).

${ }^{13}$ D. Damjanovic, Rep. Prog. Phys. 61, 1267 (1998).

${ }^{14}$ J. Macutkevic, J. Banys, and A. Matulis, Nonlinear Anal.: Modell. Control 9(1), 75 (2004).

${ }^{15}$ J. Macutkevic, S. Kamba, J. Banys, A. Brilingas, A. Pashkin, J. Petzelt, K. Bormanis, and A. Sternberg, Phys. Rev. B 74, 104106 (2006).

${ }^{16}$ D. Almond, G. K. Duncan, and A. R. West, Solid State Ionics 8, 159 (1983).

${ }^{17}$ A. Tschope, E. Sommer, and R. Birringer, Solid State Ionics 139, 255 (2001).

${ }^{18}$ T. D. Rao and S. Asthana, J. Appl. Phys. 116, 164102 (2014).

${ }^{19}$ S. S. Prosandeyev, N. M. Teslenko, and A. V. Fisenko, J. Phys.: Condens. Matter 5, 9327 (1993). 